\title{
The Application of Inertia Relief Method in Ranat and Xylophone
}

\author{
Joompon Bamrungwong ${ }^{*}$ and Pattaraweerin Woraratsoontorn \\ Department of Industrial Physics and Medical Instrumentation, King Mongkut's University of Technology North Bangkok, \\ Bangkok, Thailand \\ ${ }^{*}$ Corresponding author
}

\begin{abstract}
The percussion instrument usually involves some kind of striking. There are so many Thai percussion instrument such as the Ranat, the Cymbal ect. The aim of this analysis to study transverse modes and find out the important parameter to get proper sound frequency which are excited in bars that are struck for example the Ranat and xylophone. The theory of EulerBournoulli beam was found in textbook and applied for study the vibration of the Xylophone that the solution of governing equation need four supports thus it is impossible to find the natural frequency of the Ranat and xylophone by EulerBournoulli beam theory. In this paper uses the inertia relief method by finite element analysis to study the sound frequency of the Ranat and xylophone. The result shows that the frequency from finite element analysis is good enough for the sound analysis of percussion instrument better than Euler-Bournoulli beam theory. The arches bars are major parameter to generate sound frequency.
\end{abstract}

Keywords-sound analysis; finite element; percussion instrument; transverse mode

\section{INTRODUCTION}

The human have always struck objects to draw attention or to imbue others and themselves with rhythm. Rhythm is the basic to all forms of music in all cultures and the percussion instrument are often used to support it. The percussion instrument usually involves some kind of striking. There are so many Thai percussion instrument. The percussion instruments can be considered in three classes, the first that make use of bar for example the Ranat and xylophone. The second make use of membranes for example drums and the last make use of plates for example cymbals. The aim of this analysis to study transverse modes which are excited in bars that are struck that is the Ranat and xylophone.

\section{MATERIAL}

Thai xylophone as well known is Ranat, it is an ancient, existed since Ayutthaya era (1351 to 1767). It is a Thai musical instrument in the percussion family that consists of 21 wooden keys suspended by cords over a boat hollow shaped trough resonator and hammering by two mallets. It is used as a leading instrument in the thai orchestra in Thai Piphat ensemble. Ranat ek bars are typically made of rosewood (Dalbergia oliveri) and they are two types of Ranat ek mallets that is hard rubber mallets and soft padded mallets as show in figure 1. The hard mallets create the sharp and bright sound, normally used for faster playing. The soft mallets create the mellow and softer tone, used for slower songs. In the Thai xylophone group (Ranat group), there are several similar instrument with bars made of different types of material, such as metal and glass. Ranat Ek has a close cousin called the "Ranat thum" as show in the figure 2. The Ranat ek and Ranat thum differ in wooden keys. Its appearance is similar to the Ranat ek, but it is lower and wider. It has 18 wooden keys. It is usually played in accompaniment of a Ranat ek. The aims of this to study sound frequency of Ranat ek was being related to wooden bar geometry of the Ranat ek and support position of Ranat by finite element method. And might leads to replace wood by another material.

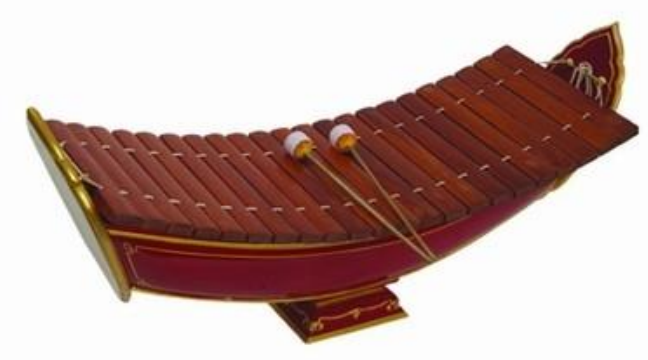

FIGURE I. RANAT EK

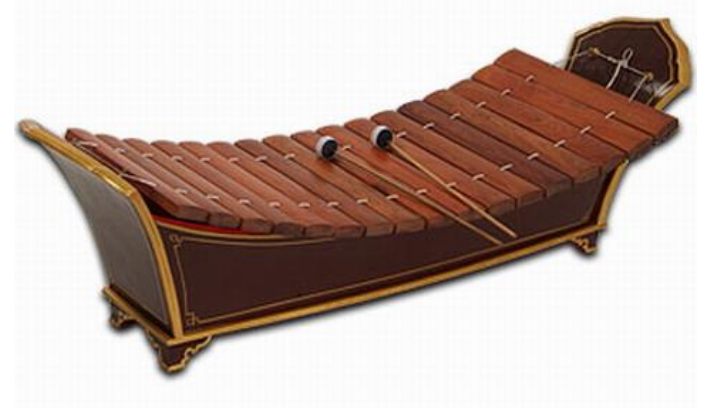

FIGURE II. RANAT THUM

Because the wooden bar in lowest frequency and next frequency has the same configuration compare with the high frequency. Thus, this study intentionally took analysis only the first wooden bar and the last wooden bar as show in figure 3 the bar without any hole for the cable support. The first wooden bar has deep arches generated in the low frequency wooden keys and the last wooden bar is shorter in length also differ arch under wooden bar. 


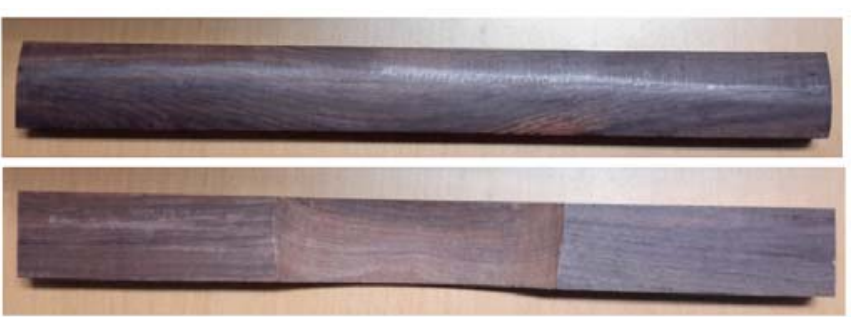

(A)

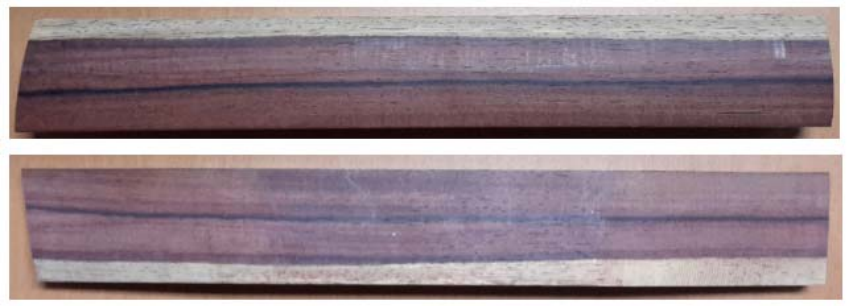

(B)

FIGURE III. THE FIRST WOODEN BAR (B) THE LAST WOODEN BAR

\section{METHOD}

The governing equation most use to explain the transverse frequency is Euler-Bournoulli beam theory [1] that start from The equations of motion of the wooden bar can be written down using Newton's second law of motion. Consider the Figure 4 shows the diagram of a wooden bar with the transverse direction of vibration that the deflection. The wooden bar no external force is applied and if $\mathrm{EI}(\mathrm{x})$ and $\mathrm{A}(\mathrm{x})$ are assumed to be constant, equation (1) simplifies so that free vibration is governed by

$$
\frac{\partial^{2} w(x, t)}{\partial t^{2}}+\frac{E I(x)}{\rho A(x)} \frac{\partial^{4} w(x, t)}{\partial x^{4}}=0
$$

the general solution of equation (1) can be calculated to be of the form

$$
X(x)=a_{1} \sin (\beta x)+a_{2} \cos (\beta x)+a_{3} \sinh (\beta x)+
$$

Then the frequency of wooden bar in hertz can be written in the form

$$
\mathrm{f}_{\mathrm{n}}=\frac{\beta_{\mathrm{n}}^{2}}{2 \pi} \sqrt{\frac{\operatorname{EI}(\mathrm{x})}{\rho \mathrm{A}(\mathrm{x})}}
$$

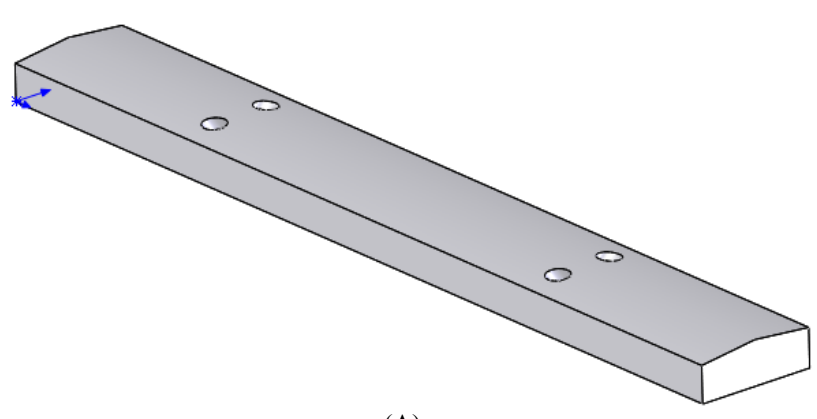

(A)
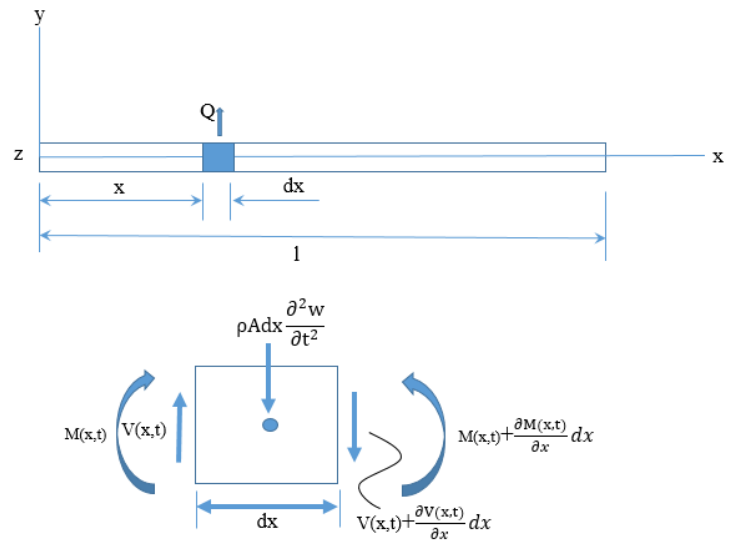

(B)

FIGURE IV. (A) WOODEN BAR (B) DIAGRAM OF AN INFINITESIMAL ELEMENT OF WOODEN BAR

The sound wave propagating through an elastic medium define as speed of sound was equal to the square root of the elastic property divided by its density, and from definition of radius of gyration the equation (3) can be written in the form

$$
\mathrm{f}_{\mathrm{n}}=\frac{\beta_{\mathrm{n}}^{2} \mathrm{R}(\mathrm{x})}{2 \pi}
$$

where

$$
\begin{gathered}
\vartheta=\sqrt{\frac{E}{\rho}} \\
R(x)=\sqrt{\frac{I(x)}{A(x)}}
\end{gathered}
$$

Noted that the natural frequency is depend on constraint of the boundary condition thus this theory is impossible to find natural frequency of the wooden bar because the wooden bar is freeing vibrate can be considered no constrain on the wooden bar. By define the wooden bar as two masses is shown in figure 5 .

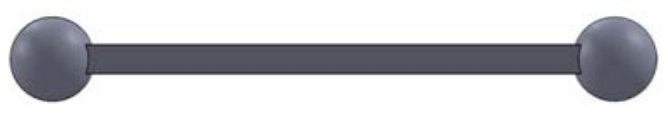

(A)

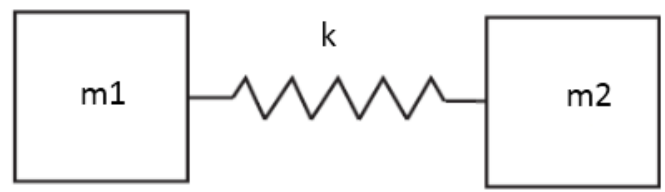

(B)

FIGURE V. (A) CONSIDER THE WOODEN BAR AS TWO MASSES AT THE END (B) SIMPLIFY THE WOODEN BAR WITH TWO MASSES TO BE SPRING TWO MASSES

The two masses ( $\mathrm{m} 1$ and $\mathrm{m} 2$ ) are connected by a spring having the stiffness $\mathrm{k}$ and there is no an external force is applied. Assume that $\mathrm{W} 1=\mathrm{W} 2$, in which $\mathrm{W} 1$ and $\mathrm{W} 2$ are the 
weight of $\mathrm{m} 1$ and $\mathrm{m} 2$, respectively. Assume the spring and two masses can only move horizontally (horizontally in Figure 5). Since no the applied force thus the system will move to left and right hand. The mass $\mathrm{m} 1$ starts moving to the right hand side compressing the spring, while the mass $\mathrm{m} 2$ is at the rest while also compressing the spring. When the spring is compressed enough to produce the reaction force more than $\mathrm{W} 1+\mathrm{W} 2, \mathrm{~m} 2$ will start moving to the right hand side. After some time, the system will arrive at a steady state, in which both $\mathrm{m} 1$ and $\mathrm{m} 2$ are moving in constant acceleration together. Considering the overall spring mass system, the equation of motion for free vibration can be written as

$$
a\left[\begin{array}{cc}
m_{1} & 0 \\
0 & m_{2}
\end{array}\right]+\left[\begin{array}{cc}
k & -k \\
-k & k
\end{array}\right]\left\{\begin{array}{l}
x_{1} \\
x_{2}
\end{array}\right\}=\left\{\begin{array}{l}
0 \\
0
\end{array}\right\}
$$

Theoretically, it is not able to determine the position of the two masses because they are not constrained in the system. Nevertheless, it can find the relative displacement by restraining either $\mathrm{x} 1$ or $\mathrm{x}_{2}$.

$$
\begin{aligned}
& \text { if } x_{1}=0 \text { then } x_{2}=\frac{a m_{1}}{k} \\
& \text { if } x_{2}=0 \text { then } x_{1}=\frac{a m_{2}}{k}
\end{aligned}
$$

This is show that the relative displacements $x_{1}-x_{2}$ is the same for either constraint. This also shows that result of the acceleration and the deformation of systems are not affected by the selection of constraints in this system it is called inertia relief analysis. Inertia load distribution relies on the assumption of constant rigid body acceleration. Inertia relief can be used in hand calculation of simple structures only. Most often, inertia relief approach is often combined with finite element analysis in the modeling and analysis of unconstrained systems. In this paper the commercial finite element packages ANSYS is used.

\section{RESULTS}

In this study, found that Euler-Bournoulli Beam Theory is impossibleto find out the frequency of the transverse wooden bar because it needs fixed support. The finite element method of unconstrained structural systems will be used to study the sound frequency of the wooden bar. Result found that the arc cut of the bottom of the wooden bar is an important parameter because if the arches were not present it is impossible to get the proper sound frequency. The deep arch in each low frequency wooden keys help the size and the cost of the Ranat within limits, which result in very long wooden keys for the low keys. The arches bar is shown in the figure 6 . The deep arches in the low frequency wooden keys decrease the wave velocity in the wooden keys. On the other hands the arches low wooden keys can be shorter than the unarches bars while still having the proper frequencies. In any wooden key the arch affects the modes of oscillation due to the wave speed is low at the thin center of the wooden key than at the thick ends, the wave length of a standing wave of a certain frequency is shorter in the middle of the wooden key than at the ends. Another important of the arch under wooden key concerns tuning and tone quality.

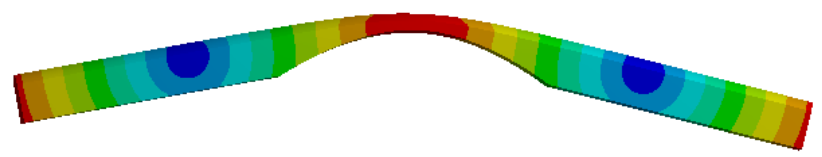

(A)

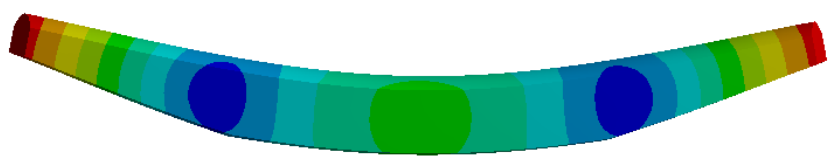

(B)

FIGURE VI. EXAGGERATED (A) SIMPLE BENDING MODE IN A FIRST WOOD KEY (B) SIMPLE BENDING MODE IN A LAST WOOD KEY

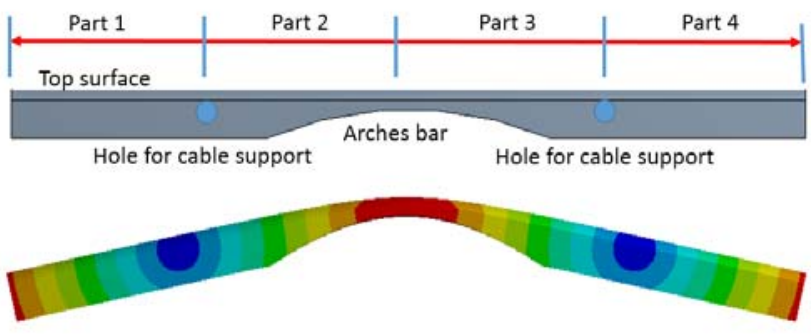

FIGURE VII. THE WOODEN BAR CAN BE DIVIDED INTO FOUR PARTS

From the study by simulation also found that there are two points are quite not changed positioning thus the wooden bar can be defined the support at the blue color as shown in the figure 7 author recommends the support should be closed to the top surface of the Ranat. Practically, a musician needs "KaSuan" is for draft to get the size of the all wooden key since the first key to the last key. Ka-Suan has size 39-29 is meant the first wooden key has long $39 \mathrm{~cm}$ and in steps for next wooden key until the last wooden key has long $29 \mathrm{~cm}$. However, it depends on a place of the musician. By this study the Ranat can be standardized by define Ka-Suan which detailed the cable for support, arches bars.

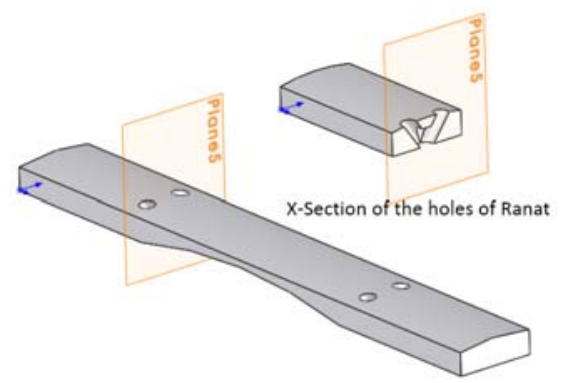

FIGURE VIII. THE WOODEN BAR FO RANAT

\section{CONCLUSION}

The sound frequency of the percussion instrument such as the Xylophone or the Ranat (Thai percussion instruments) has the transverse modes generated in bending mode, without the arches curve it is impossible to get proper sound frequency. The application of inertia relief method by finite element analysis is suitable for analysis percussion analysisalso revealed that the wooden bar can be divided into four parts the 
first part and the last part is for cable support and should be closed to the top surface of the wooden bar as shown in the figure 7. The characteristic of the wooden bar is shown in the figure 6 (A) the first wooden bar (B) the last wooden bar. By this approach might leads to complex design of percussion instruments.It also can be noted that the Xylophone needs two hole for cable support at beside of the wooden bar while the Ranat has three hole for each support one bigger hole is at the bottom of the wooden bar and two hole like a "V" shaped at the top surface of Ranat that point to the bottom hole as shown in the figure 8 . However, the holes on the wooden key are not significance for the sound frequency but the positioning of the hole is significance.

\section{REFERENCES}

[1] Davis R Lapp, "Building a copper pipe Xylophone" from www.iop.org/journals/physed

[2] Percussion instrument [On-line]. Jan, 09, 2016, available: https:/ccrma.stanford.edu/CCRMA/Courses/152/percussion.html

[3] Richard E. Berg, David G. Stork,"The Physics of Sound" Second Edition, Prentice Hall,1995

[4] B. Joompon,"Analysis and Design The Sound Frequency of a Treble

[5] Gamelan Using FEA" MENETT\#20, THAILAND, 2000.

[6] Inman, D. J. (1994) “Engineering vibration”, Prentic-Halll, Inc., New York.

[7] Rossing, T. D. (1982) The science of sound, Addison-Wesley Publishing Company,Inc., London.

[8] Ranat [online]. Feb20,2016, available: https://en.wikipedia.org/ wiki/Ranat. 